\title{
A Design of Spatial XQuery for Mobile and Location-Based Applications*
}

\author{
Soon-Young Park ${ }^{1}$, Jae-Dong Lee ${ }^{2}$, and Hae-Young Bae ${ }^{1}$ \\ ${ }^{1}$ Dept. of Computer Science and Information Engineering, Inha University \\ Yonghyun-dong, Nam-gu, Inchon, 402-751, Korea \\ sunny@dblab.inha.ac.kr, hybae@inha.ac.kr \\ ${ }^{2}$ Dept. of Information and Computer Science, Dankook University \\ Hannam-ro, Yongsan-gu, Seoul, 140-714, Korea \\ letsdoit@dku.edu
}

\begin{abstract}
In this paper S-XML (Spatial-eXtensible Markup Language) is proposed for mobile and location-based applications. And in order to handle effectively the extension, a Spatial XQuery language and its processing modules has been designed. Because our work is based on a spatial DBMS, the Spatial XQuery statements are firstly translated into Spatial SQL statements. By working on an existing spatial DBMS, we can use its existing functions such as query optimization, spatial indexes, concurrency control mechanism, and recovery scheme. Translation of the Spatial XQuery into SQL has been explained using examples. Because the results from the spatial database system are in the form of tables, we again need to translate the results into S-XML statements. A working example of the proposed system as an Emergency Support System is also presented. Prospected application areas of the proposed system are almost all mobile and location-based systems such as m-commerce, ubiquitous systems in mobile environments.
\end{abstract}

\section{Introduction}

Recently the uses of mobile and location-based applications are popularized and extended. And requirements to geographic information systems also have been changed. Geography Markup Language (GML) [9] is the active standard for exchanging spatial data on the Internet and mobile devices, but it is limited only for text and graphic data of static geographic information. Location-based Services (LBS) can be provided as more attractive services when they utilize tracking information of moving objects. It would be very convenient if a car navigation system provides voice mode as well as the basic screen mode.

In this paper, S-XML (Spatial-eXtensible Markup Language) is proposed, which extends the GML by adding three schemas: voice schema, tracking schema, and POI (Point Of Interest). In order to handle the S-XML data effectively, a spatial XQuery language has been designed. In this research, the geographic data are stored in a spatial database management system and the spatial XQuery constructs submitted by users are translated into spatial SQL and evaluated by the SDBMS(Spatial Database Management System). The SDBMS supports the query optimization methods and concurrency control for processing multiple queries simultaneously.

* This research was supported by the MIC (Ministry of Information and Communication), Korea, under the ITRC (Information Technology Research Center) support program supervised by the IITA (Institute of Information Technology Assessment) 
The spatial XQuery supports basic operations (e.g., =, <, and >), spatial operations (e.g., sp_disjoint, sp_intersects, and sp_contains), and operations for moving objects (e.g., mo_after, mo_before, mo_first, and mo_last). With these spatial operations and the operations for moving objects, the spatial XQuery can be effectively used on LBS (Location-Based Services) platforms. The spatial XQuery also supports the VOICE tag, using which the direction information can be provided by voice mode interface.

This paper is organized as follows. Section 2 summarizes the various approaches to handle XML queries. Section 3 presents the information model of S-XML and the syntax and semantics of spatial XQuery. Section 4 describes the overall processing of spatial XQuery. Spatial XQueries are first translated into equivalent spatial SQL statements and then evaluated by database management system with spatial operation. Section 5 provides a working scenario of using the spatial XQuery. In this scenario, the user first locates the nearest hospital from an accident and the driver of an ambulance from the hospital gets the direction to the accident site when the car approaches crossroads. Section 6 summarizes the contributions of the paper.

\section{Related Work}

Two research groups have contributed to the developments of XML query languages. First, the document research group has their experience in designing languages and tools for processing structured documents. Several query languages have been proposed for XML. For example, XQuery uses XPath in order to support operations on document path expressions. XQuery offers very powerful query capabilities. But XQuery does not provide much support for querying spatial elements. It simply treats spatial elements as numerical values [8]. Second, the database research group has their experience in designing query language and processors for data-oriented computations. XQuery also uses the basic structure of SQL language for relational databases [13].

In database approaches, XML documents are stored in preexisting database systems $[1,4,9,10]$ and the XML queries are translated into SQL or other database languages [6, 12]. The problems of updating XML data stored in tables [11] and indexing XML data [3, 7] have also been presented. Prototypes such as SilkRoute [5, 6] and XPERANTO [2] have proposed algorithms for efficiently encoding relational data as XML documents.

Consequently, database approaches make good use of the existing functions of database systems, which include query optimization, indexes, concurrency control, and recovery. On the other hand, native approaches are more customized for XML documents. Because huge amount of geographic information is already stored in spatial database systems, we take the database approach. By using the spatial XQuery language, the user can access not only the extended spatial information but also the ordinary text and graphic information in terms of XML forms.

\section{S-XML and Spatial XQuery}

In this section, the structure of S-XML is introduced at first and then the syntax and semantics of Spatial XQuery are proposed with some examples. 


\subsection{S-XML}

S-XML is based on GML 3.0 and is designed to provide effective location based services to the user in mobile environments. S-XML consists of three main schemas, i.e., voice schema, tracking schema, and POI schema.

\section{(a) Voice Schema}

In mobile environments, voice information is often more useful than text or graphic information especially when the user is driving a car. To be effective, the grammar of the voice information is designed as simple as possible (see Fig. 1). The voice information can be either location information or turn information.

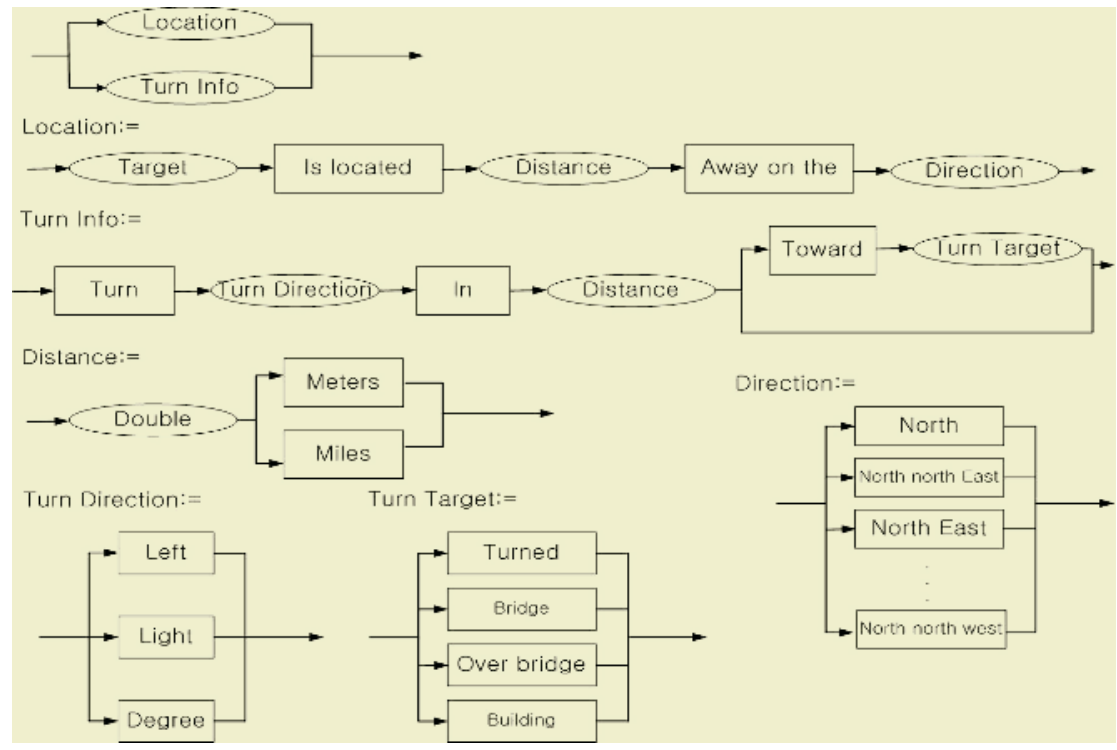

Fig. 1. Grammar of Voice Schema

\section{(b) POI Schema}

POI schema includes the location information of objects in specific area, the optimal path to destination, the type of moving objects (e.g., car, human, subway, etc.), information of the source and the destination, information of representative objects near the destination, and additional information about the destination (e.g., phone number and email address). Fig. 2 shows a part of POI schema, which includes a starting point, a target point and a route.

\section{(c) Tracking Schema}

Tracking schema specifies the changes of status of specific object as time changes. The information model includes the type of moving objects, information of specific points and the time when the point passed, and information of direction and velocity. Fig. 3 shows a part of Tracking Schema, which specifies the locus type. A locus consists of a series of points, time, location and direction. 


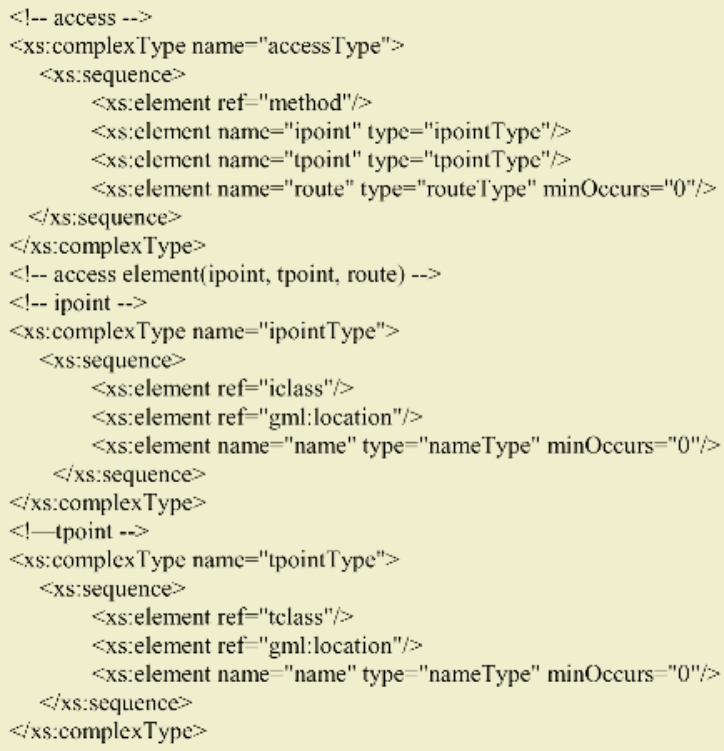

Fig. 2. Part of POI Schema that includes the specification of starting and target points

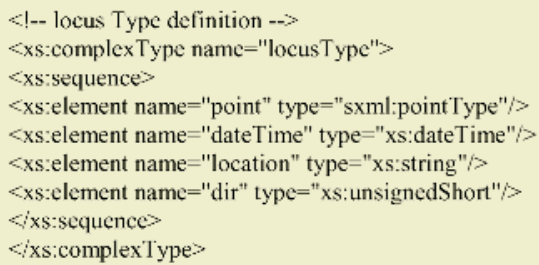

Fig. 3. Part of Tracking Schema that includes the specification of locus

\subsection{Spatial XQuery}

Spatial XQuery follows basic FLWR (For Let Where Return) structure of XQuery with path expressions so that SQL users feel easy to use and the elements and attributes of S-XML can be effectively accessed. Spatial XQuery also supports the spatial data, spatial relational operators, and spatial functions that are recommended by OGC specifications [9]. In Spatial XQuery, spatial operators and spatial functions have 'sp' as a prefix in their names. Functions of moving objects have 'mo' as a prefix in their names. A list of operators and functions specified in Spatial XQuery are as follows.

(a) Basic Operator for Spatial Objects

sp_Dimension (g Geometry): int, sp_GeometryType (g Geometry): string sp_AsText (g Geometry): string, sp_AsBinary (g Geometry): binary sp_IsEmpty (g Geometry): int, sp_IsSimple (g Geometry): int sp_Boundary (g Geometry): geometry, sp_Envelop (g Geometry): geometry 
(b) Functions of Point Type

sp_x (p Point): double, sp_y (p Point): double

(c) Functions of Curve Type

sp_StartPoint(c Curve): point, sp_EndPoint(c Curve): point

sp_IsClosed(c Curve,): int, sp_IsRing(c Curve): int, sp_Length(c Curve): double

(d) Functions of LineString Type

sp_NumPoints(1 LineString): int, sp_PointN(1 LineString, $n$ Int): point

(e) Functions of Surface Type

sp_Centroid (s Surface): point, sp_PointOnSurface(s Surface): point, sp_Area(s Surface): double

(f) Functions of Polygon Type

$\mathrm{sp} \_$ExteriorRing(p polygon): linestring, sp_InteriorRingN(p polygon, $\mathrm{n}$ int): linestring

sp_NumInteriorRing(p polygon): int

(g) Functions of GeometryCollection Type

sp_NumGeometries(g GeomCollection): int

sp_GeometryN(g GeomCollection, $\mathrm{n}$ int): geometry

(h) Functions of MultiCurve Type

sp_IsClosed(mc MultiCurve,): int, sp_Length(mc MultiCurve): double

(i) Topology Operators between two spatial objects

sp_Equals(g1 Geometry, g2 Geometry): int, sp_Disjoint(g1 Geometry, g2 Geometry): int

sp_Intersects(g1 Geometry, g2 Geometry): int, sp_Touches(g1 Geometry, g2 Geometry): int

sp_Overlaps(g1 Geometry, g2 Geometry) int, sp_Crosses(g1 Geometry, g2 Geometry): int

sp_Within(g1 Geometry, g2 Geometry)): int, sp_Contains(g1 Geometry, g2 Geometry): int

(j) Functions of Distance Relationship

sp_Distance(g1 Geometry, g2 Geometry): double

(k) Functions of Spatial Operations

sp_Intersection(g1 Geometry, g2 Geometry):geometry

sp_Difference(g1 Geometry, g2 Geometry): geometry

sp_Union(g1 Geometry, g2 Geometry): geometry

sp_Symdifference(g1 Geometry, g2 Geometry): geometry

sp_Buffer(g1 Geometry, d Double precision): geometry, sp_Convexhull(g Geometry): geometry

(1) Functions of Moving Objects

mo_First(p Position, $n$ Int): point, mo_Last(p Position, $n$ Int): point

mo_After(p Position, $t$ Time): point, mo_Before(p Position, $t$ Time): point

mo_Snapshot(p Position, $t$ Time): point, mo_Snapshot(p Position, g Geometry): time

mo_Slice(p Position, pe Period(start, end)): Linestring

mo_Slice(p Position, g Geometry): Linestring

mo_Project(p Position): Linestring, mo_Project(pe Period(start, end, g Geometry): point

(m) Functions of Voice Service

POIDirection(p Position): string, ShortestPathDirection(p Position, i Int): string

Details of XQuery syntax are not discussed in this paper. The meaning of Spatial XQuery will be clear from the following example.

Example 1. This query retrieves the names of cities that are located within $5 \mathrm{~km}$ from any river in rivers. $x m l$.

for \$a in document("cities.xml”) //city

$\$ b$ in document("rivers.xml") //river

where sp_overlap $(\$ a / o b j$, sp_buffer $(\$ b / o b j, 5000))$ eq true

return $\quad<$ Within5000CityName $>\$$ a/name $<$ /Within5000CityName $>$ 


\section{Processing of Spatial XQuery}

The query processor of Spatial XQuery consists of Parser, SQL Info Generator, Result Info Generator, and SQL Translator (see Fig. 4). The queries from the user interface are first parsed to generate parse trees, which are sent to SQL Info Generator and Result Info Generator. Spatial XQueries are finally translated into spatial SQL, based on the information generated by SQL Info Generator and Result Info Generator. SQL Info Generator analyzes the clauses FOR, LET, and WHERE from the Spatial XQuery statements and generates a mapping table for query translation. The RETURN clause of Spatial XQuery is analyzed by Result Info Generator and a linked list of tags and elements is generated.

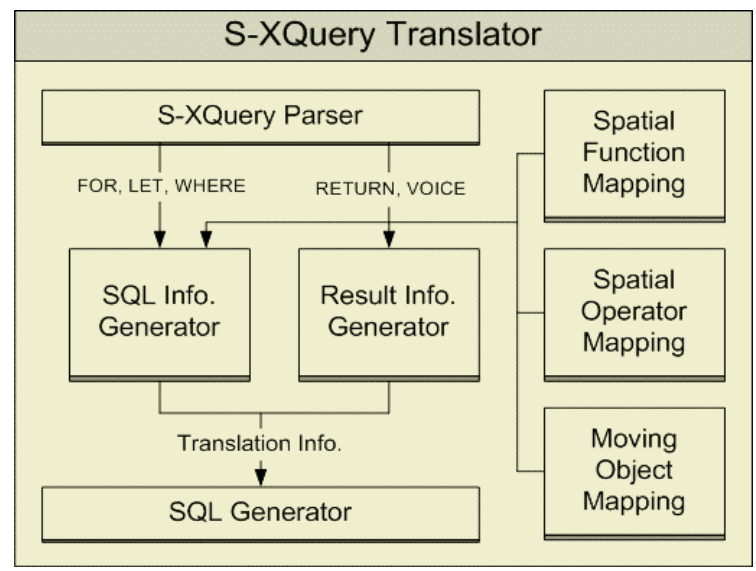

Fig. 4. Structure of the Query Processor of Spatial XQuery

Details of the procedure of translating Spatial XQuery are presented using an example. Consider the query in Example 1. By analyzing the FOR and WHERE clauses, we can generate the mapping table as in Table 1. In Table 1, tag names and variable names are translated into corresponding table names in spatial database. Operators in Spatial XQuery are also mapped into the corresponding ones in spatial database management system. Such mapping information is provided by Spatial Operator Mapping which is the Mapping Module of Spatial Operators. In this example, the spatial operator of Spatial XQuery $s p \_o v e r l a p$ in mapped into operator overlap in spatial database management system.

Table 1. Mapping from Spatial XQuery to Spatial SQL

\begin{tabular}{|l|l|l|}
\hline & \multicolumn{1}{|c|}{ Spatial XQuery } & \multicolumn{1}{c|}{ Spatial SQL } \\
\hline From & $\begin{array}{l}\text { Cities.xml//City } \\
\text { Rivers.xml//River }\end{array}$ & $\begin{array}{l}\text { City } \\
\text { River }\end{array}$ \\
\hline Select & \$a/name & City.name \\
\hline Where & $\begin{array}{l}\text { sp_overlap }(\$ a / o b j, \\
\text { Buffer(\$b/obj, 5000) }) \text { eq true }\end{array}$ & $\begin{array}{l}\text { overlap(City.obj, Buffer(River.obj, } \\
\text { 5000) }=\text { true }\end{array}$ \\
\hline Order by & NULL & NULL \\
\hline
\end{tabular}



lows.

Based on the mapping table in Table 1, spatial SQL query can be generated as fol-

Select City.name

From City, River

Where overlap(City.obj, Buffer(River.obj, 5000)) = true;

The results from SQL Info Generator and Result Info Generator can be represented as a graph. The graph in Fig. 5 is generated for this example, which includes nodes for start tag, table name, column name and end tag.

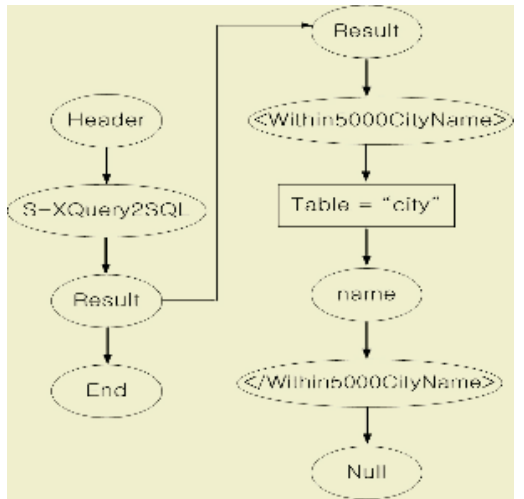

Fig. 5. Graphical Representation of results from SQL Info Generator and Result Info Generator

Using the results in Fig. 5 and the table returned as a result for the spatial SQL statement, the following S-XML document will be generated for this example.

$<$ Within5000CityName $>$

$<$ name $>$ Han River </name $>$

$</$ Within5000CityName $>$

\section{Working Scenario}

A scenario to apply Spatial XQuery in LBS environments is discussed in this section. This is an example of Emergency Support System that includes POI Service and Shortest Path Service. Suppose that a car accident takes place and there are some injuries (see Fig. 6). The user of this system is looking for the nearest hospital and wants an ambulance for injured people. Once the nearest hospital is identified by the Emergency Support System, the hospital is requested to dispatch an ambulance to the accident place. Then the driver of the ambulance asks the direction to the accident place while he drives.

Fig. 7 is a Spatial XQuery that looks for the nearest hospital from the accident place. The coordinates of the accident place are available from a GPS.

Fig. 8 is the spatial SQL query that is translated from the Spatial XQuery in Fig. 7. The spatial operator sp_distance is translated into spatial database management operator distance and for optimization the selection condition is included in the WHERE clause. 


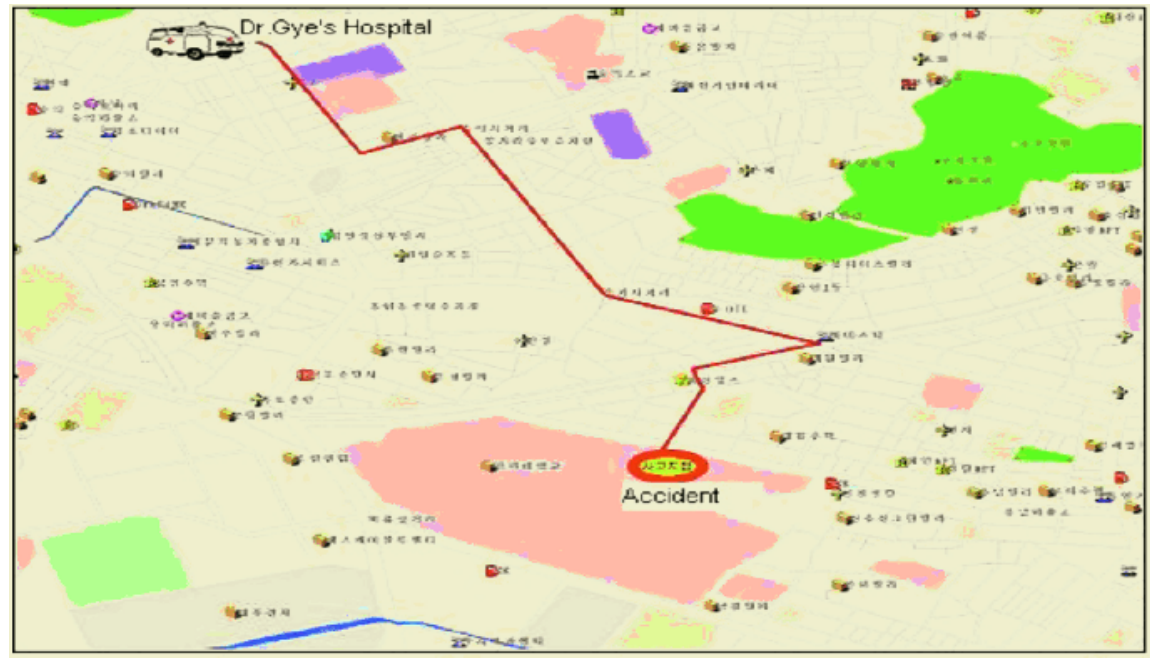

Fig. 6. Car Accident and Ambulance from the Nearest Hospital

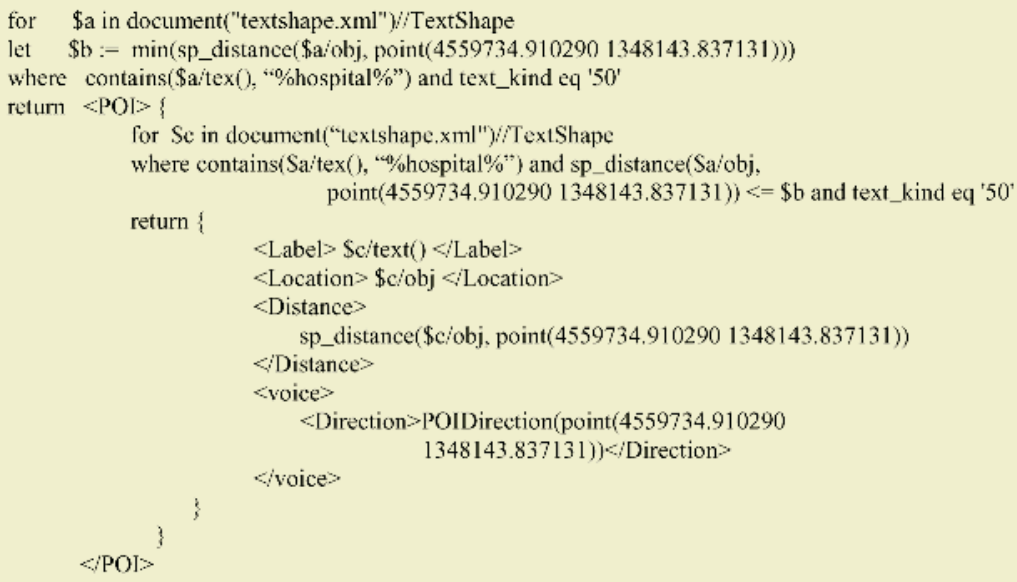

Fig. 7. Spatial XQuery looking for the Nearest Hospital

select text, obj, distance(obj, point(4559734.9102901348143.837131))

from textshape

where text like "\%hospital\%' and distance(obj, point(4559734.910290 1348143.837131)) $<=$ (select min(distance( obj, point(4559734.910290 1348143.837131) )) from textshape where text like '\%hospital $\%$ ' ) and text_kind - '50';

Fig. 8. Spatial SQL that is translated from the Spatial XQuery in Fig. 7

From the result returned for the spatial SQL query in Fig. 8, an S-XML document is generated as in Fig. 9. In Fig. 9, a hospital named "Dr. Gye's Hospital" is selected and the distance and direction to it are included. 


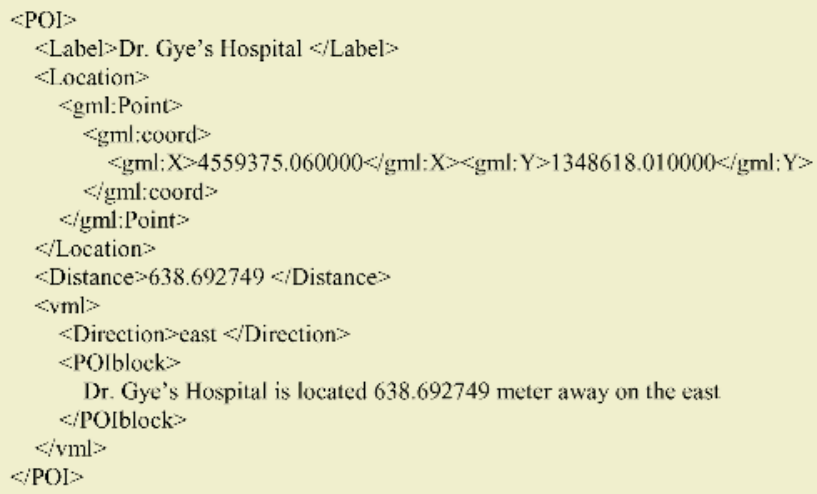

Fig. 9. The Nearest Hospital and its Distance and Direction from the Accident Place

\section{Conclusion}

S-XML is proposed for mobile and location-based applications. In order to handle effectively the extension, we have designed a Spatial XQuery language and its processing modules. Because our work is based on a spatial database system, the Spatial XQuery statements are first translated into spatial SQL statements. By working on existing spatial database system, we can use the existing functions of database systems such as query optimization, spatial indexes, concurrency control, and crash recovery. Translation of the Spatial XQuery into spatial SQL has been explained using an example.

Application areas of the proposed system are almost all mobile and location-based systems such as LBS, ubiquitous systems, and distributed control and management systems. In order to be more powerful, it needs to be extended with various development tools. Naive users may have difficulties to program their queries using Spatial XQuery. Some user-friendly designed GUI tools could make the system more effective. Even current execution model allows multiple queries; we need to devise more sophisticated optimization strategies for multiple queries.

\section{References}

1. P. Bohannon, J. Freire, P. Roy, and J. Simeon, "From XML schema to relations: A costbased approach to XML storage," Proceedings of IEEE International Conference on Data Engineering (ICDE), 2002.

2. M. Carey et al., "XPERANTO: Publishing Object-Relational Data as XML," Proceedings of Workshops on Web and Databases (WebDB), 2000.

3. B. Cooper et al., "A Fast Index for Semistructured Data," Proceedings of International Conference on Very Large Databases (VLDB), 2001.

4. A. Deutsch, M. Fernandez, and D. Suciu, "Storing semistructured data with STORED," Proceedings of the ACM SIGMOD International Conference on Management of Data (SIGMOD), 1999.

5. M. Fernandez et al., "Publishing Relational Data as XML: The SilkRoute Approach," IEEE Data Engineering Bulletin, 24(2), 2001. 
6. M. Fernandex, Y. Kadiyska, A. Morishima, D. Suciu, and W. Tan, "SilkRoute: A framework for publishing relational data in XML," ACM Transactions on Database Systems, 2002.

7. D. Kha, M. Yoshikawa, and S. Uemura, "An XML Indexing Structure with Relative Region Coordinate," Proceedings of IEEE International Conference on Data Engineering (ICDE), 2001.

8. Z. Liu, E.-P. Lim, W.-K. Ng and D. H. Goh, On Querying Geospatial and Georeferenced Metadata Resources in G-Portal, Proceedings of Joint Conference on Digital Libraries (JCDL), 2003.

9. OGC, Geography Markup Language (GML) Implementation Specification 3.0, 2003.

10. S.-Y. Park, J.-D. Lee, and H.-Y. Bae, "Easily Accessible GML-based Geographic Information System for Multiple Data Server over the Web," Proceedings of International Conference on Information System Technology and its Applications (ISTA), 2003.

11. I. Tatarinov, Z. Ives, A. Halevy, and D. Weld, "Updating XML," Proceedings of ACM SIGMOD International Conference on Management of Data (SIGMOD), 2001.

12. I. Tatarinov, S. Viglas, K. Beyer, J. Shanmugasundaram, E. Shekita, and C. Zhang, "Storing and querying ordered XML using a relational database system," Proceedings of ACM SIGMOD International Conference on Management of Data (SIGMOD), 2002.

13. W3C, XQuery 1.0: An XML Query Language, 2002. 